\title{
Insights into the mechanisms of splicing: more lessons from the ribosome
}

\author{
Maria M. Konarska ${ }^{1,3}$ and Charles C. Query ${ }^{2}$ \\ ${ }^{1}$ The Rockefeller University, New York, New York 10021, USA; ${ }^{2}$ Department of Cell Biology, Albert Einstein College of \\ Medicine, Bronx, New York 10461, USA
}

Detailed analyses of spliceosome complexes have revealed an impressive complexity of composition, with five snRNAs and several-hundred protein factors involved in their formation (e.g., Fromont-Racine et al. 2000; Makarov et al. 2002; Zhou et al. 2002; for review, see Burge et al. 1999; Jurica and Moore 2003). Not only is the spliceosome composed of a large number of factors, but formation of the catalytically competent complex requires multiple rearrangements of its five snRNP subunits, achieved through a series of assembly steps involving changes in both the composition of associated factors and their conformations within the complex (for review, see Nilsen 1998; Staley and Guthrie 1998). This changing structural complexity of the spliceosome reflects underlying mechanistic dynamics throughout the entire splicing process.

Indeed, although the spliceosome has long been recognized to be highly dynamic, this was generally regarded as a property of its assembly, and rarely considered in models of the catalytic phase of the reaction. Splicing catalysis proceeds through two consecutive transesterifications involving three sites of the intron; in the first reaction, the branch site nucleophilically attacks the 5'SS, producing a lariat intermediate and cleaved 5' exon, and in the second reaction, the $5^{\prime}$ exon attacks the 3'SS, yielding spliced mRNA and lariat intron products. We have recently proposed a dynamic model of the catalytic spliceosome in which conformations of the complex supporting the two catalytic steps are in kinetic competition, with modulation of the stability of the first- and second- step conformations resulting in improvement of one of the catalytic steps to the detriment of the other (Query and Konarska 2004). In a recent issue of Genes \& Development, Villa and Guthrie (2005) present new data in support of rearrangements of the spliceosome between the two catalytic steps.

\section{The two-state model}

What is the evidence that the two catalytic steps are associated with two different conformations of the spli-

${ }^{3}$ Corresponding author.

E-MAIL konarsk@mail.rockefeller.edu; FAX (212) 327-7147.

Article and publication are at http://www.genesdev.org/cgi/doi/10.1101/ gad. 1363105 ceosome? Considering that the substrates for the two chemical reactions are different, some rearrangement of the substrate(s) and/or enzyme at the catalytic center is logically required. If the spliceosome uses a single active site for both catalytic steps, lariat intermediate formed in the first step would need to be displaced to allow positioning of the 3'SS for the second step (Steitz and Steitz 1993); furthermore, stereochemical analysis of splicing is consistent with some rearrangement being required between the two steps (Moore and Sharp 1993). In this case, the spliceosome would exist in two distinct conformational states during the catalytic phase, binding the substrates differently for the two steps. Consistent with this notion, a large number of mutations in spliceosomal factors (Prp8, Prp16, U6 snRNA, Isy1) alter the relative efficiencies of the first and second steps. For spliceosomal mutants that suppress second-step defects, improvement of second-step efficiency is accompanied by inhibition of the first step. Likewise, suppressors of firststep defects inhibit the $2^{\text {nd }}$ step. The fact that improvement of one step is linked to inhibition of the other strongly argues for competition between mutually exclusive spliceosome conformations for the two steps. Mutations in many different spliceosomal factors have similar effects on the splicing of substrates carrying different intron mutations (located either at the 5'SS, branch site, or $\left.3^{\prime} \mathrm{SS}\right)$, arguing for a general mode of action. This is likely achieved through global stabilization of one spliceosome conformation relative to the other through alteration of interdomain contacts within the complex (Fig. 1).

Among the spliceosomal factors implicated in the modulation of these conformations are U6 snRNA, Prp16, and Prp8, all thought to be involved in direct interactions with the catalytic center, and Isy1, a nonessential component of the NTC (Prp NineTeen Complex). U6 is thought to be the key active-site component and positions the 5'SS for the first step (for review, see Villa et al. 2002). Prp8 is a large, highly conserved protein component of U5 snRNP implicated in interactions with multiple spliceosomal components and all three intron sites involved in catalysis (for review, see Grainger and Beggs 2005). Prp16 is a DEAH-box ATPase thought to facilitate the transition between the first and second 
Figure 1. Scheme for progression of the pre-mRNA splicing process, highlighting the Prp16-dependent transition between the two catalytic steps. The first and second catalytic steps require different conformations of the spliceosome, the equilibrium between these conformations being modulated by interactions of Prp16, Isy1, Prp8, and U6 snRNA. As indicated by the green and red arrows, certain alleles of these factors improve the first step and inhibit the second, and other alleles act oppositely. The Prp16 ATPase facilitates the transition between the first and second steps and, as a result, provides an oppor tunity for rejection of substrates that do not efficiently proceed to the second step (i.e., discard). This modulation of transition and opportunity for discard is likely repeated at multiple points in both assembly and post-catalytic phases.

steps of splicing, and was initially identified in a screen for mutants with reduced fidelity of splicing (Couto et al. 1987; Burgess et al. 1990; Schwer and Guthrie 1991). The NTC is a heteromeric protein complex present in the catalytically active spliceosome that contributes to stability of spliceosomal interactions including 5'SS interaction with U6 (Makarov et al. 2002; Chan et al. 2003; Makarova et al. 2004; Chan and Cheng 2005). The recent data of Villa and Guthrie (2005) demonstrate an antagonistic relationship between Prp16 and the NTC-component Isy1. Mutant prp16 alleles are defective for the second step of splicing, and this defect is relieved by deletion of ISY1 both in vivo and in vitro; likewise, deletion of ISY1 rescues the cold-sensitive phenotype of a prp16 allele in vivo. isy1 deletion alone inhibits the first step of splicing. These results argue that Isyl stabilizes the firststep conformation and its absence facilitates transition to the second-step conformation. These data add to the argument that Prp16 facilitates the transition to the second step, with its mutant alleles stabilizing the first-step conformation (Fig. 1).

Additional factors will certainly be added to the above list of modulators of the first-to-second-step transition, with U2 snRNA and some other components of the NTC exemplifying likely candidates. Furthermore, a variety of pre-mRNA mutations are predicted to affect the equilibrium between spliceosomal conformations through stabilization/destabilization of interactions between the substrate for the first and the second step and components of the catalytic center.

Thus, the emerging picture is consistent with the structure of the catalytic center being affected by several adjacent components connected through a network of interactions. Specifically, genetic interactions of Prp16 with U6, U6 with Prp8, Prp8 with Prp16, Prp16 with Isy1, and Isy1 with U6 have been demonstrated (Madhani and Guthrie 1994; McPheeters 1996; Collins and Guthrie 1999; Query and Konarska 2004; Villa and Guthrie 2005). In earlier static models of the catalytic spliceosome, such complexity of genetic interactions was frequently interpreted as indicative of stable physical interactions. According to the dynamic equilibrium model, these components need not physically interact, although such direct interactions are certainly possible. Instead, either destabilization or hyperstabilization of in- teractions affecting the structure of the catalytic center (these may include interactions remote from the catalytic center itself) would alter the equilibrium between spliceosomal conformations by globally altering the stability of one conformation relative to the other.

\section{Analogies between splicing and ribosomal decoding}

The ribosome offers an attractive model to illustrate the effects of alterations of equilibrium between conformations. tRNA decoding involves transitions between open and closed conformations at the $30 \mathrm{~S} \mathrm{~A}$ site that can be modulated by the stability of interface contacts /Ogle et al. 2002). Numerous similarities between the ribosome and the spliceosome have previously been noted (e.g., Sharp 1981; Guthrie 1991; Fabrizio et al. 1997), and recent high-resolution structural information combined with detailed functional analysis of the ribosome provides insights into the dynamics of rearrangements in a large RNP complex (for review, see Rodnina et al. 2002, 2005; Cochella and Green 2005; Ogle and Ramakrishnan 2005). An essential aspect of the decoding process is the pairing of an mRNA codon with its cognate amino acyltRNA anti-codon delivered to the ribosome in a ternary complex with GTP and EF-Tu. Correct pairing provides additional contacts with components of the A site, which stabilize both domain closure at the A site and the correctly paired codon-anticodon through an induced fit mechanism. Modulation of equilibrium between open and closed conformations explains how many disparate mutations in the 30S and 50S subunits, tRNA, and the GTPase EF-Tu affect the fidelity of translation (Ogle et al. 2003). For example, mutations in ribosomal proteins S4 and S5, located away from the A site, destabilize S4:S5 interface contacts that normally stabilize the open conformation, thus favoring domain closure at the A site. Multiple antibiotics and certain mutations in S12 may also affect the equilibrium of open/closed conformations in a similar way. Facilitation of 305 domain closure, even in the presence of incorrect anticodon-codon pairing leads to reduced fidelity, whereas impairment of domain closure leads to increased fidelity during translation. Domain closure, and accompanying changes in the structures of the $50 \mathrm{~S}$ subunit, aa-tRNA, and EF-Tu activate GTP hydrolysis by EF-Tu. Collectively, these 
changes result in the transmission of a signal from the A site, where codon-anticodon interaction is recognized, to the peptidyl transferase site in the 50S subunit, where catalysis occurs.

Although equivalent models for the spliceosome are not available, the mechanistic similarities between the ribosome and spliceosome may provide useful new insights into the splicing process. Whereas the specifics of the reactions catalyzed by these RNPs are clearly different, some fundamental generalities of how macromolecular complexes function will certainly apply to both.

\section{Toward an integrated model of spliceosome function}

The two-state model of spliceosome function has already helped to explain many otherwise paradoxical observations. Thus far, it has provided a unified explanation for the effects of mutations in various spliceosomal components on splicing of disparate mutant substrates. However, the model's main utility may still lie in the future, as it allows for formulation of new questions and testable predictions concerning basic aspects of the mechanism of splicing. Here we suggest some such predictions and questions, and speculate on their possible answers. We anticipate that future efforts in the field will vindicate some of these predictions and disprove others.

\section{Molecular description of the first-to-second-step transition}

Based on accumulated genetic and biochemical data, several models of the spliceosome catalytic center have been proposed, in which a network of conserved RNARNA pairing interactions involving snRNAs and premRNA implicate U2 and U6 snRNAs in critical contacts with the splice sites and branch site (for review, see Nilsen 1998; Burge et al. 1999; Collins and Guthrie 2000). The 5'SS is held and positioned for first-step catalysis, in part by base-pairing interactions with the universally conserved ACAGAG region of U6 snRNA, and the region surrounding the branch site is paired with the conserved GUAGUA sequence of U2 snRNA. In addition, U5 snRNA interacts with both exons, contributing to their positioning for catalysis (for review, see Newman 1997). However, apart from this latter interaction, little is known about substrate positioning for secondstep catalysis.

If the catalytic phase of the reaction involves a conformational rearrangement between the first and second steps, what is the molecular nature of this rearrangement? If Prp16 facilitates the transition between the two catalytic steps, what are the direct consequences of its action? Can the ribosome offer any insights into mechanistic interpretations of this transition?

First, what specifically needs to happen during this transition? If the spliceosome acts like most other multistep enzymes, using a single catalytic center to carry out sequential reactions, its catalytic center would need to rearrange or "open" to unlock the first-step products and allow them to reposition. The lariat intermediate formed in the first step would need to be removed from the catalytic center and replaced by the 3'SS (the secondstep substrate). Subsequent closure of the catalytic center would allow second-step catalysis. An important question is how these events are allocated into kinetically separable steps. A temporal map may be attainable from analysis of the effects of genetic interactions between components of the catalytic center (including the substrate) on the splicing of substrates that are rate-limiting for a particular step in the multistep kinetic scheme. For example, two factor mutations that affect the same kinetic step in opposing directions should cancel each other's effects (e.g., one suppressor allele improving opening of the catalytic center and another inhibiting it). However, if these mutations affect kinetically distinct steps, then their combination would be expected to yield an overall improvement of splicing (e.g., a combination of first-step suppressor alleles that inhibit opening of the catalytic center with second-step suppressor alleles that improve substrate repositioning). This property can be used to identify and distinguish between separate kinetic steps in vivo. Although available genetic systems allow for significant further analysis in this direction, the development of more precise biochemical systems will inevitably be required for detailed kinetic description of these processes.

What is the consequence of Prp16 action? This ATPase is known to act at the transition between first and second steps (Schwer and Guthrie 1991). However, the observed improvement of the first step by prp16 alleles defective for this transition is consistent with Prp16 acting either to open the catalytic center or to reposition the substrate (Query and Konarska 2004; Villa and Guthrie 2005). The effects of combining suppressor alleles of prp8, prp16, and U6 snRNA argue that Prp16 and U6 affect different kinetic events from those affected by prp8 alleles (Query and Konarska 2004). In contrast, the observation that prp16 mutants and isy1 deletion cancel each other's effects suggests that they may affect the same kinetic event (Villa and Guthrie 2005). Further studies suggest that the effects of prp16 and U6 alleles on this transition precede the effects of prp 8 alleles, consistent with the idea that Prp16 facilitates an unlocking step that occurs prior to repositioning of substrate (L. Liu, C. Query, and M. Konarska, unpubl.).

By analogy to the detailed kinetic description of rearrangement events during tRNA decoding by the ribosome, we propose a tentative kinetic framework for the spliceosome first-to-second-step transition. As proposed previously, Prp16 may be thought of as analogous to EF$\mathrm{Tu}$ (Burgess et al. 1990). Closure of the first-step catalytic center would be expected to be induced by correct positioning of the first-step substrates; this closure would provide the necessary environment for first-step catalysis. By analogy to the activation of EF-Tu GTPase by $30 \mathrm{~S}$ domain closure and distortion in tRNA structure, it is possible that Prp16 ATPase is activated by a closed catalytic center and perhaps also by the branched structure of the lariat intermediate. The resulting ATP hydrolysis by 
Prp16 would result in its dissociation, facilitating opening of the catalytic center, and thus "unlocking" the first-step products for subsequent repositioning.

This view of conformational changes associated with this and other analogous ATP-dependent steps, suggests that the importance of identifying "direct targets" of the ATPases is diminished relative to understanding their consequences on the structure of the complex as a whole, and that the important structural alteration effected within the spliceosome may be displaced by a large distance from the actual location of the ATPase.

\section{Possible models of the second-step catalytic center}

The notion that lariat intermediate formed in the first step is removed from the first-step catalytic center during the transition to the second step (M.M. Konarska, J. Vilardell, and C.C. Query, in prep.) has several implications for the arrangement of the enzyme and substrates during the second step. The branch region is thought to base pair to U2 snRNA during the first step, and thus, the question arises as to whether this pairing is disrupted for the second step, or whether the entire branch structure paired to $\mathrm{U} 2$ is removed from the catalytic center. In the latter case, a different region of U2 snRNA would be present at the catalytic center, thus altering its composition and properties. This could contribute to the binding of different substrates (i.e., 3'SS) for the second step. Because very little is known of the arrangement of the second-step catalytic center, future work will need to resolve a number of important issues as follows: What is the fate of the branch site:U2 pairing? How is the 3'SS positioned relative to the 5' exon, U2, U5, and U6 snRNAs? Where is the 3'SS moved from? Is the 5'SS repositioned between the two steps? Does the moved lariat structure contribute to second-step catalysis? Is the structure assumed by the second-step substrate (3'SS) similar to that of the first-step product (lariat intermediate)?

\section{Substrate selectivity of splicing}

What is responsible for the observed fidelity of splicing? In mammalian systems, multiple examples of regulatory proteins point to assembly (for review, see Maniatis and Tasic 2002; Black 2003). In contrast, genetic analyses in yeast have identified factors involved in the catalytic phase (Burgess et al. 1990; Umen and Guthrie 1995, 1996; Collins and Guthrie 1999; Siatecka et al. 1999; Query and Konarska 2004). How does the mammalian spliceosome catalyze the reactions on substrates that deviate substantially from the consensus and thus must be defective/suboptimal in binding to the catalytic center? Is the role of regulators of alternative splicing restricted to modulation of spliceosome assembly or do they have further roles in affecting the catalytic steps?

In previous models, increased stability of interactions at the catalytic center was generally expected to improve the reaction. Thus, predictions for splice-site selection were based on the calculated stability of $5^{\prime}$ SS pairing to U1 or U6 snRNAs (e.g., Libri et al. 2002; Roca et al. 2005). In contrast, the two-state spliceosome model predicts that a number of splice-site and branch-site mutations would alter the stability of substrate binding at the catalytic center, improving one step, but inhibiting the other. Indeed, extended 5'SS pairing to U1 results in inhibition of splicing (Nelson and Green 1990; Staley and Guthrie 1998) and extended pairing to U6 inhibits the transition to the second step (M.M. Konarska, J. Vilardell, and C.C. Query, in prep). Similar logic applies to other steps in the process, including both assembly and release phases, as the states flanking each spliceosomal transition are likely to be in competition.

Suboptimal sites (i.e., sites that deviate from the consensus) are a hallmark of alternatively spliced premRNAs (Fairbrother et al. 2002). Such suboptimal sites are utilized less efficiently, not only because of defective recognition during assembly, but also because they are less well bound at the catalytic center, may activate ATPases less efficiently, and may also be defective for repositioning during the first-to-second-step transition. Whereas it is possible that wild-type substrates induce rapid progression through spliceosomal transitions, precluding the establishment of equilibria, the use of suboptimal substrates makes it more likely that some transitions are inefficient, and thus, that the preceding steps may reach thermodynamic equilibrium. In such a scenario, these transitions could be modulated by extrinsic factors that alter these equilibria. Regulation of alternative splicing certainly involves modulation of splice-site selection during spliceosome assembly through interactions of protein factors. However, to produce functional mRNA, such suboptimal sites must also subsequently be bound at the catalytic center and undergo catalysis. Alteration of the equilibrium between the two catalytic steps can improve catalysis on such suboptimal sites. In fact, mutations in several splicing factors (i.e., first- and second-step suppressor alleles) do alter splicing efficiency of numerous suboptimal splice sites /Query and Konarska 2004). It is tempting to speculate that similar effects on the use of suboptimal sites may also be achieved through association of alternative splicing factors, resulting in the modulation of the efficiencies of the two catalytic steps. Detailed biochemical studies will be required to demonstrate such a suppressor activity of alternative splicing factors.

\section{Proofreading mechanisms and discard pathways}

More than a decade ago, kinetic proofreading was proposed as a strategy used to reduce incorrect mRNA splicing (Burgess and Guthrie 1993). As discussed above, this model is reminiscent of translation. Indeed, PRP16 was first identified in a screen for mutants with reduced fidelity of splicing, suggesting a similarity of function to ribosomal EF-Tu (Couto et al. 1987). The correlation between reduced ATPase activity and improved splicing of mutant substrates by the selected prp16 alleles suggested that ATP hydrolysis by Prp16 is required to achieve fi- 
delity of splicing (Burgess et al. 1990). In this model, the fidelity of the second step is modulated by the relative rates of two Prp16-dependent reactions coupled with ATP hydrolysis, i.e., conformational change in the spliceosome that allows progression to the second step, and discard of mutant lariat intermediates from the first-step catalytic center. Thus, the discard pathway would be in kinetic competition with productive splicing.

Insights into the kinetic pathways of splicing raise further questions regarding the detailed mechanisms of discard. Is discard a consequence of the substrate being stalled at a specific stage, e.g., being released from the binding site for one step, but unable to interact with the next step binding site? By analogy to the ribosomal process, proofreading by Prp16 would arise from the slow rate of repositioning of the lariat intermediate. After ATP hydrolysis, Prp16 dissociates from the spliceosome (Schwer and Guthrie 1991; Villa and Guthrie 2005), potentially opening or loosening contacts within the catalytic center and allowing for repositioning of the "unlocked' lariat intermediate to the second step catalytic center. Once "unlocked", lariat intermediate may be only weakly bound to the spliceosome, permitting kinetic competition between discard and productive repositioning. Productive repositioning must involve binding of 3'SS and the lariat structure to their second-step binding sites; inefficient binding at either of these sites might be predicted also to result in discard, by analogy to inefficient initial binding of tRNA-EF-Tu-GTP ternary complex at the 30S A site. Thus, mutants in spliceosomal factors that affect repositioning would be predicted either to increase discard (e.g., alleles of prp8 that suppress first-step defects, thought to inhibit or slow repositioning) or to reduce it (e.g., isy1 deletion or prp 8 alleles that suppress second-step defects, thought to facilitate repositioning).

Is discard limited to rejection of "incorrect" lariat intermediates that underwent the first step of splicing, or can substrates be rejected at multiple stages in the process at various transitions in the reaction? As previously proposed on theoretical grounds, each ATPase-associated step may offer an opportunity for kinetic discrimination of "incorrect" substrates (Burgess and Guthrie 1993). In the repetitive steps of ATP hydrolyses in the splicing pathway, the ATPase acting after Prp16 is Prp22; thus, the next opportunity for discard would be after its action. For wild-type substrates, this would represent the release of spliced mRNA; whereas for substrates inefficient for second-step catalysis, this would allow for discard of the unreacted intermediates. Discard at this and other steps would be expected to be demonstrable using substrates limiting for the transition facilitated by the ATPase in question. Indeed, recent evidence suggests that such kinetic discrimination does occur during Prp22-dependent exit from the second step (J. Staley, pers. comm.).

Is discard during the catalytic phase a consequence of conformational transitions that occur without the substrate having undergone catalysis? For example, are unreacted pre-mRNAs (that were slow for first-step cataly- sis) discarded in transition out of the first-step conformation? During the noncatalytic phases of assembly, discard could result from failure of various binding events that, in a productive pathway, must occur prior to ATP hydrolysis. Which substrate defects would be susceptible to discard? Different intron mutants are differentially discarded (Bousquet-Antonelli et al. 2000; Hilleren and Parker 2003). Detailed analysis of the discard pathways of such substrates may yield important information with regard to the mechanism not only of discard processes, but also of the transitions associated with the productive splicing process.

\section{Conclusions}

Previous models of ATPases controlling consecutive transitions during spliceosomal assembly combined with recent impressively detailed structural and mechanistic studies of the ribosome, have resulted in the formulation of a substantially more detailed model of spliceosome function. We hope that the above speculations and predictions will stimulate further work on unresolved mechanistic issues, as we are convinced that a detailed understanding of the mechanism is necessary for ultimate explanation of all aspects of the splicing process, from its evolutionary origins to the regulation of alternative splicing.

\section{Acknowledgments}

We are grateful to Rachel Green, Beate Schwer, and Duncan Smith for helpful discussions and critical readings of the manuscript. This work was supported by NIH grants GM49044 to M.M.K. and GM57829 to C.C.Q.

\section{References}

Black, D.L. 2003. Mechanisms of alternative pre-messenger RNA splicing. Annu. Rev. Biochem. 72: 291-336.

Bousquet-Antonelli, C., Presutti, C., and Tollervey, D. 2000. Identification of a regulated pathway for nuclear pre-mRNA turnover. Cell 102: 765-775.

Burge, C.B., Tuschl, T.H., and Sharp, P.A. 1999. Splicing of precursors to mRNAs by the spliceosomes. In The RNA world, 2nd ed. (eds. R.F. Gesteland et al.), pp. 525-560. Cold Spring Harbor Laboratory Press, Cold Spring Harbor, New York.

Burgess, S.M. and Guthrie, C. 1993. Beat the clock: Paradigms for NTPases in the maintenance of biological fidelity. Trends Biochem. Sci. 18: 381-384.

Burgess, S., Couto, J.R., and Guthrie, C. 1990. A putative ATP binding protein influences the fidelity of branchpoint recognition in yeast splicing. Cell 60: 705-717.

Chan, S.-P. and Cheng, S.-C. 2005. The Prp19-associated complex is required for specifying interactions of U5 and U6 with Pre-mRNA during spliceosome activation. J. Biol. Chem. M505060200.

Chan, S.P., Kao, D.I., Tsai, W.Y., and Cheng, S.C. 2003. The Prp19p-associated complex in spliceosome activation. Science 302: 279-282.

Cochella, L. and Green, R. 2005. Fidelity in protein synthesis. Curr. Biol. 15: R536-R540.

Collins, C.A. and Guthrie, C. 1999. Allele-specific genetic in- 
teractions between Prp8 and RNA active site residues suggest a function for Prp8 at the catalytic core of the spliceosome. Genes \& Dev. 13: 1970-1982.

- 2000. The question remains: Is the spliceosome a ribozyme? Nat. Struct. Biol. 7: 850-854.

Couto, J.R., Tamm, J., Parker, R., and Guthrie, C. 1987. A transacting suppressor restores splicing of a yeast intron with a branch point mutation. Genes \& Dev. 1: 445-455.

Fabrizio, P., Laggerbauer, B., Lauber, J., Lane, W.S., and Lührmann, R. 1997. An evolutionarily conserved U5 snRNP-specific protein is a GTP-binding factor closely related to the ribosomal translocase EF-2. EMBO J. 16: 4092-4106.

Fairbrother, W.G., Yeh, R.F., Sharp, P.A., and Burge, C.B. 2002. Predictive identification of exonic splicing enhancers in human genes. Science 297: 1007-1013.

Fromont-Racine, M., Mayes, A.E., Brunet-Simon, A., Rain, J.C., Colley, A., Dix, I., Decourty, L., Joly, N., Ricard, F., Beggs, J.D., et al. 2000. Genome-wide protein interaction screens reveal functional networks involving Sm-like proteins. Yeast 17: 95-110.

Grainger, R.J. and Beggs, J.D. 2005. Prp8 protein: At the heart of the spliceosome. RNA 11 533-557.

Guthrie, C. 1991. Messenger RNA splicing in yeast: Clues to why the spliceosome is a ribonucleoprotein. Science 253: $157-163$.

Hilleren, P.J. and Parker, R. 2003. Cytoplasmic degradation of splice-defective pre-mRNAs and intermediates. Mol. Cell 12: 1453-1465.

Jurica, M.S. and Moore, M.J. 2003. Pre-mRNA splicing: Awash in a sea of proteins. Mol. Cell 12: 5-14.

Libri, D., Duconge, F., Levy, L., and Vinauger, M. 2002. A role for the Psi-U mismatch in the recognition of the 5' splice site of yeast introns by the U1 small nuclear ribonucleoprotein particle. J. Biol. Chem. 277: 18173-18181.

Madhani, H.D. and Guthrie, C. 1994. Genetic interactions between the yeast RNA helicase homolog Prp16 and spliceosomal snRNAs identify candidate ligands for the Prp16 RNA-dependent ATPase. Genetics 137: 677-687.

Makarov, E.M., Makarova, O.V., Urlaub, H., Gentzel, M., Will, C.L., Wilm, M., and Lührmann, R. 2002. Small nuclear ribonucleoprotein remodeling during catalytic activation of the spliceosome. Science 298: 2205-2208.

Makarova, O.V., Makarov, E.M., Urlaub, H., Will, C.L., Gentzel, M., Wilm, M., and Lührmann, R. 2004. A subset of human 35S U5 proteins, including Prp19, function prior to catalytic step 1 of splicing. EMBO I. 23: 2381-2391.

Maniatis, T. and Tasic, B. 2002. Alternative pre-mRNA splicing and proteome expansion in metazoans. Nature 418: 236-243.

McPheeters, D.S. 1996. Interactions of the yeast U6 RNA with the pre-mRNA branch site. RNA 2: 1110-1123.

Moore, M.J. and Sharp, P.A. 1993. Evidence for two active sites in the spliceosome provided by stereochemistry of premRNA splicing. Nature 365: 364-368.

Nelson, K.K. and Green, M.R. 1990. Mechanism for cryptic splice site activation during pre-mRNA splicing. Proc. Nat1. Acad. Sci. 87: 6253-6257.

Newman, A.J. 1997. The role of U5 snRNP in pre-mRNA splicing. $E M B O T$. 16: 5797-5800.

Nilsen, T.W. 1998. RNA-RNA interactions in nuclear premRNA splicing. In RNA structure and function (eds. R.W. Simons and M. Grunberg-Manago), pp. 279-307. Cold Spring Harbor Laboratory Press, Cold Spring Harbor, New York.

Ogle, J.M. and Ramakrishnan, V. 2005. Structural insights into translational fidelity. Annu. Rev. Biochem. 74: 129-177.

Ogle, J.M., Murphy, F.V., Tarry, M.J., and Ramakrishnan, V.
2002. Selection of tRNA by the ribosome requires a transition from an open to a closed form. Cell 11: 721-732.

Ogle, J.M., Carter, A.P., and Ramakrishnan, V. 2003. Insights into the decoding mechanism from recent ribosome structures. Trends Biochem. Sci. 28: 259-266.

Query, C.C. and Konarska, M.M. 2004. Suppression of multiple substrate mutations by spliceosomal prp 8 alleles suggests functional correlations with ribosomal ambiguity mutants. Mol. Cell 14: 343-354.

Roca, X., Sachidanandam, R., and Krainer, A.R. 2005. Determinants of the inherent strength of human $5^{\prime}$ splice sites. RNA 11: 683-698.

Rodnina, M.V., Daviter, T., Gromadski, K., and Wintermeyer, W. 2002. Structural dynamics of ribosomal RNA during decoding on the ribosome. Biochimie 84: 745-754.

Rodnina, M.V., Gromadski, K.B., Kothe, U., and Wieden, H.J. 2005. Recognition and selection of tRNA in translation. FEBS Lett. 579: 938-942.

Schwer, B. and Guthrie, C. 1991. PRP16 is an RNA-dependent ATPase that interacts transiently with the spliceosome. $\mathrm{Na}$ ture 349: 494-499.

Sharp, P.A. 1981. Speculations on RNA splicing. Cell 23: $643-$ 646.

Siatecka, M., Reyes, J.L., and Konarska, M.M. 1999. Functional interactions of Prp8 with both splice sites at the spliceosomal catalytic center. Genes \& Dev. 13: 1983-1993.

Staley, J.P. and Guthrie, C. 1998. Mechanical devices of the spliceosome: Motors, clocks, springs, and things. Cell 92: 315-326.

Steitz, T.A. and Steitz, J.A. 1993. A general two-metal-ion mechanism for catalytic RNA. Proc. Natl. Acad. Sci. 90: 6498-6502.

Umen, J.G. and Guthrie, C. 1995. A novel role for a U5 snRNP protein in 3' splice site selection. Genes \& Dev. 9: 855-868.

-1996. Mutagenesis of the yeast gene PRP8 reveals domains governing the specificity and fidelity of 3 ' splice site selection. Genetics 143: 723-739.

Villa, T. and Guthrie, C. 2005. The Isylp component of the NineTeen Complex interacts with the ATPase Prp16p with to regulate the fidelity of pre-mRNA splicing. Genes \& Dev. 19: 1894-1904.

Villa, T., Pleiss, J.A., and Guthrie, C. 2002. Spliceosomal snRNAs: $\mathrm{Mg}^{2+}$-dependent chemistry at the catalytic core? Cell 109: 149-152.

Zhou, Z., Licklider, L.J., Gygi, S.P., and Reed, R. 2002. Comprehensive proteomic analysis of the human spliceosome. $\mathrm{Na}$ ture 419: 182-185. 


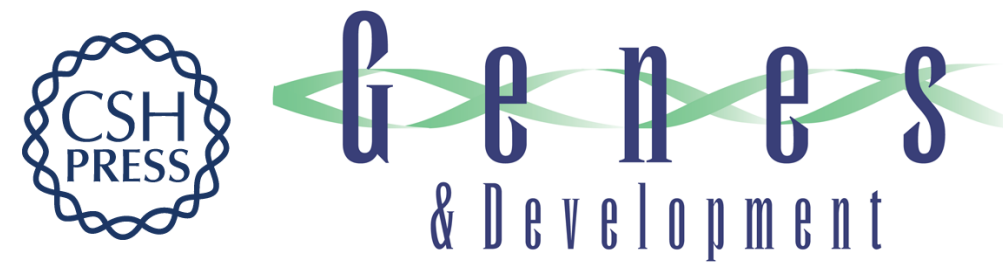

\title{
Insights into the mechanisms of splicing: more lessons from the ribosome
}

\author{
Maria M. Konarska and Charles C. Query
}

Genes Dev. 2005, 19:

Access the most recent version at doi:10.1101/gad.1363105

$\begin{array}{ll}\text { References } & \begin{array}{l}\text { This article cites } 41 \text { articles, } 19 \text { of which can be accessed free at: } \\ \text { http://genesdev.cshlp.org/content/19/19/2255.full.html\#ref-list-1 }\end{array}\end{array}$

License

Email Alerting Receive free email alerts when new articles cite this article - sign up in the box at the top Service right corner of the article or click here.

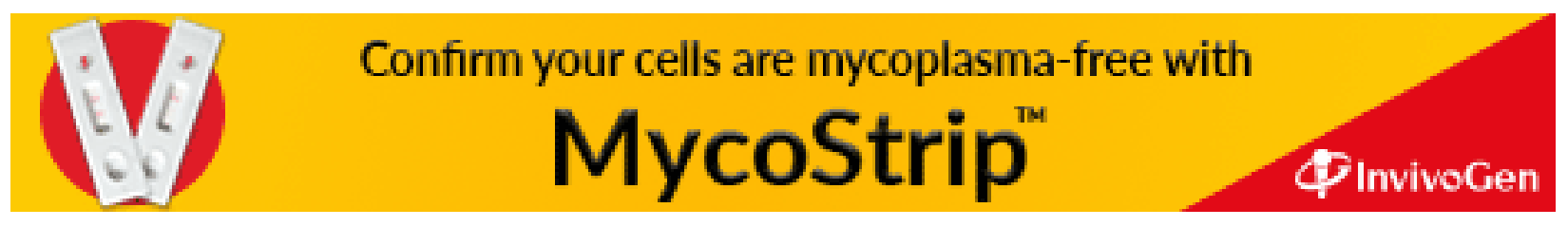

